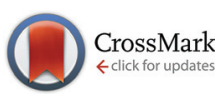

Cite this: Org. Biomol. Chem., 2016, 14,5529

Received 20th January 2016, Accepted 2nd February 2016

DOI: 10.1039/c6ob00168h

www.rsc.org/obc

\section{Comparison of boron-assisted oxime and hydrazone formations leads to the discovery of a fluorogenic variant $\dagger$}

\author{
Cedric J. Stress, Pascal J. Schmidt and Dennis. G. Gillingham*
}

\begin{abstract}
We use kinetic data, photophysical properties, and mechanistic analyses to compare recently developed high-rate constant oxime and hydrazone formations. We show that when Schiff base formation between aldehydes and arylhydrazines is carried out with an appropriately positioned boron atom, then aromatic B-N heterocycles form irreversibly. These consist of an extended aromatic structure amenable to the tailoring of specific properties such as reaction rate and fluorescence. The reactions work best in neutral aqueous buffer and can be designed to be fluorogenic - properties which are particularly interesting in bioconjugation.
\end{abstract}

\section{Introduction}

Condensation of aldehydes or ketones with $\alpha$-effect nucleophiles such as hydroxylamines and hydrazines is an important ligation reaction in chemical biology. ${ }^{1-3}$ Reports from us ${ }^{4}$ and others ${ }^{5,6}$ have shown that proximal functional groups enhance the rate of oxime/hydrazone condensation at neutral $\mathrm{pH}$. Recent work from our group has demonstrated that proximal boronic acids increase the rate of oxime condensation up to more than $10^{4} \mathrm{M}^{-1} \mathrm{~s}^{-1}$ under neutral aqueous conditions. ${ }^{7}$ Nevertheless, oximes and hydrazones are unstable to hydrolysis under aqueous conditions. ${ }^{8}$ We have quantified the reversibility of aldoximes that bear proximal boronic acids and found a stability on the order of several hours in neutral aqueous buffer; others have found that the reversibility problem is particularly acute with ketoximes, ${ }^{9}$ which equilibrate in minutes. While reversibility may be beneficial for certain applications,${ }^{10}$ a durable link is often required for biological studies ${ }^{11}$ or in practical applications such as for antibody-drug conjugates. ${ }^{12}$

In the search for an irreversible variant of an oxime or hydrazone condensation we turned to hydrazines as $\alpha$-effect

Department of Chemistry, University of Basel, St. Johanns-Ring 19, 4056 Basel, Switzerland.E-mail: dennis.gillingham@unibas.ch

$\dagger$ Electronic supplementary information (ESI) available. CCDC 1448404. For ESI and crystallographic data in CIF or other electronic format see DOI: 10.1039/ c6ob00168h nucleophiles. The inspiration for this choice came from two reports, published more than fifty years ago, demonstrating that with hydrazines the initial Schiff base adducts (structure 3 in Fig. 1) undergo a secondary intramolecular cyclization to deliver aromatic, boron-substituted isoquinoline derivatives, which were stable to prolonged boiling in acid or base. ${ }^{13,14}$ These reports on 4,3-borazaroisoquinolines (BIQs) were largely forgotten until the 1990s when reports from Groziak $^{15}$ structurally confirmed and extended ${ }^{16}$ the earlier findings. The Groziak group also proposed these materials as the basis of unique pharmacophores. ${ }^{17,18}$ Indeed several BIQs have shown growth inhibitory activity against different types of bacteria. ${ }^{18-20}$ In a very recent development, the Bane lab has shown that BIQ formation works well under aqueous conditions and could be used for bioconjugation. ${ }^{21}$ In this work we present a mechanistic analysis and comparison of oxime, hydrazone, and BIQ formation (i.e. structures 1, 2, 3, and 4 in Fig. 1). The data should serve as a guidebook to potential users for determining which conjugation is best suited to their specific application. Furthermore, we demonstrate that when the aromatic system of the BIQ is extended and appropriately substituted to deliver a push-pull system, then a fluorogenic reaction is possible. Turn-on fluorescence is important in

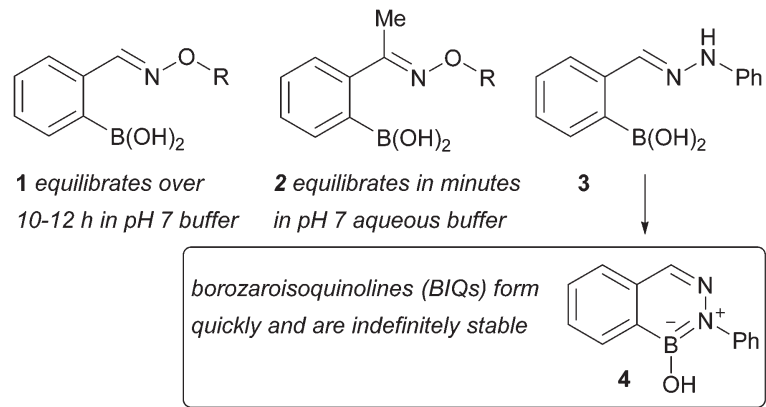

Fig. 1 Boron accelerates the formation of Schiff bases of type 1, 2, and 3 from carbonyl compounds and $\alpha$-effect amines; 3, however, engages in a secondary reaction that leads to the stable BIQ 4. 
cellular assays since it reduces background during imaging and can remove the need for washing steps. ${ }^{22,23}$

\section{Results and discussion}

We have shown recently that boronic acid groups in ortho position to an aldehyde greatly enhance the rate of oxime formation (see a in Fig. 2). ${ }^{7}$ We hypothesize that this is due to the formation of a five-membered ring intermediate prior to the normally rate-limiting dehydration step (see b in Fig. 2). This intermediate must eliminate a boronate rather than a water molecule to deliver the oxime - a process that should be far more facile since protonation of the cyclic boronate would occur right around neutral $\mathrm{pH} .{ }^{24-26}$ This mechanistic pathway is supported by a previous study which examined the effect of boron on labelling the $\varepsilon$-amino group of lysines. ${ }^{27}$ The overall oxime formation is too rapid for us to detect intermediates so

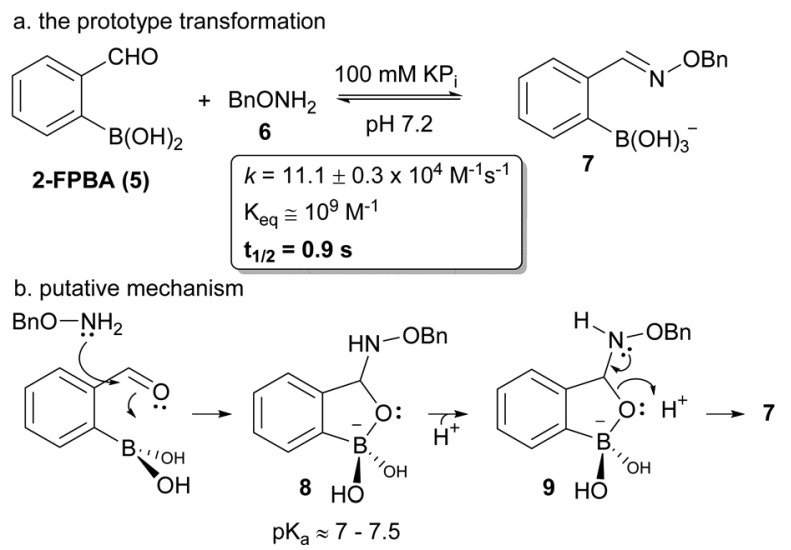

c. an 8-membered ring does not promote efficient coupling
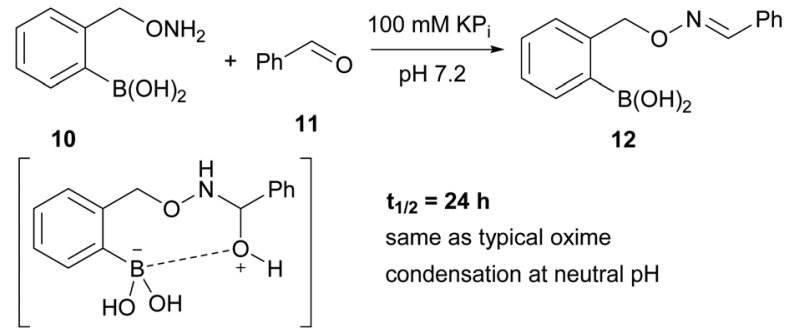

d. boronic acids that lack an open coordination site react slower

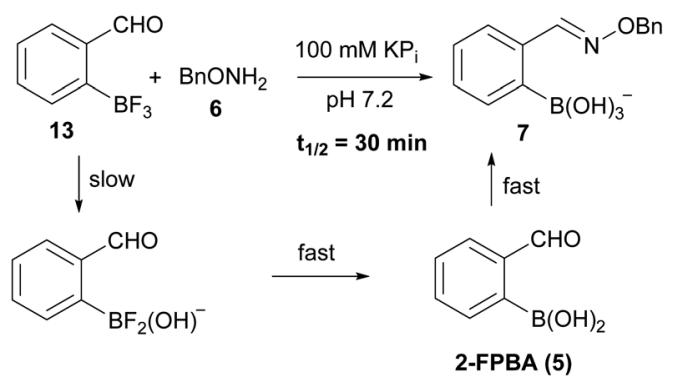

Fig. 2 The prototype boron assisted oxime condensation (a), our working mechanistic hypothesis (b) and substrate variations that provide some insight on the mechanism (c \& d). we turned to substrate variation to probe this mechanistic hypothesis. We had already shown ${ }^{7}$ that aldehyde positioning relative to the boronic acid is important for high reaction rates, with only ortho-positioning leading to any acceleration. para- or meta-Formylphenylboronic acids led to oxime condensations with rates comparable to benzaldehyde. The new substrates added in Fig. 2 (see c \& d) are also consistent with the mechanistic hypothesis. The condensation of $\mathbf{1 0}$ and $\mathbf{1 1}$ (see c in Fig. 2) would require the formation of an intermediate 8-membered ring if the boronic acid were involved. The rate of this transformation is comparable to substrates that bear no boronic acid and hence a small ring size seems crucial. Our attempts to look at other ring sizes were thwarted by synthetic difficulties. An important aspect of the proposed mechanism is the direct involvement of boron. 2-Formylphenyltrifluoroborate 13 maintains the boron, but does not have an open coordination site. Treatment of $\mathbf{1 3}$ with 6 (see d in Fig. 2) delivers product far slower than 2-FPBA but still faster than benzaldehyde $\left(t_{1 / 2}=48 \mathrm{~h}\right.$ at $1 \mathrm{mM}$; Note: extrapolated from four data points). In addition the hydrolyzed molecule 7 is the only observed product (i.e. no fluoroborates); hence in this case hydrolysis of the trifluoroborate must occur before condensation. Indeed the rate of oxime formation is consistent with rates of hydrolysis for related aryltrifluoroborates (see the ESI page $18 \dagger$ for an additional example with a coordinatively saturated arylboronate ester). ${ }^{28}$

In our previous report we found that aldoximes are reversible but took several hours until the equilibrium was reached. ${ }^{7}$ To further investigate the influence of substituents on the stability and reversibility of oximes we treated ketoxime 14 with a five-fold excess of 2-formylphenylboronic acid (2-FPBA). NMR analysis indicated rapid equilibration and a strong energetic preference for the aldoxime: after the first measurement (15 min) about $60 \%$ of the ketoxime was converted to the corresponding aldoxime, and after 75 minutes only the aldoxime was observed (compare time points in Fig. 3). A substantial reduction in transition state energy for formyl versus acetyl Schiff bases has also been seen by others ${ }^{9}$ and is likely a result of the preference for the ketoximine nitrogen to bind to the boron in an iminoboronate interaction, thus activating it for hydrolysis; X-Ray crystallography indicates no such interaction in the ground-state for the aldoxime (see B in Fig. 3). We speculate that the energetic reason for this difference is related to Raine's hypothesis that the basicity of the nitrogen of the Schiff base is decisive in determining stability of oximes and hydrazones. The additional $\sigma$-donation of the methyl substituent should render its nitrogen more basic (compare $\mathrm{X}=$ Me to $\mathrm{X}=\mathrm{H}$ in panel c, Fig. 3) and therefore more amenable to protonation or interaction with a Lewis acid.

In the search for an irreversible bioconjugation we turned to arylhydrazines. Although hydrazones (such as 16 in Fig. 4) are known to be more labile than oximes, ${ }^{8}$ reports from the 1960s showed that hydrazones with a boronic acid group in ortho position undergo an intramolecular cyclization reaction to 4,3-borazaroisoquinolines (BIQ). ${ }^{13,14}$ The Bane lab has very recently described that this reaction could offer a powerful tool 
A<smiles>COc1ccccc1C(=NOC(C)c1ccccc1)[N+](=O)[O-]</smiles>

2-FPBA (5) 14

B

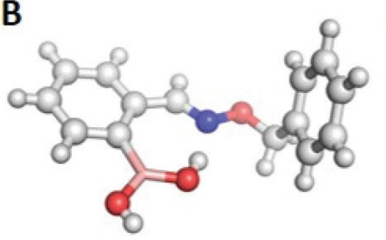

C<smiles>O=Cc1ccccc1OCCOc1ccccc1O</smiles><smiles>CC(O/N=C/c1ccccc1O)c1ccccc1</smiles>
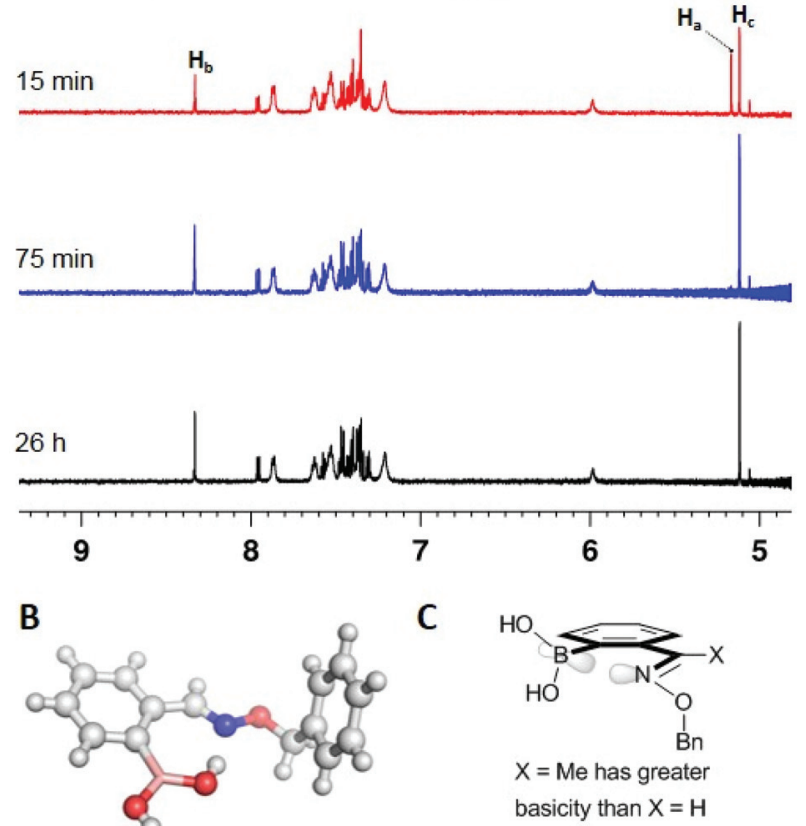

Fig. 3 A. NMR indicates rapid equilibration and a strong preference for 7 over 14. B. X-Ray analysis of compound 7 suggests no iminoboronate in the ground state. $C$. The increased $\sigma$-donation of methyl versus hydrogen likely increases basicity and hence reduces stability of oximes.
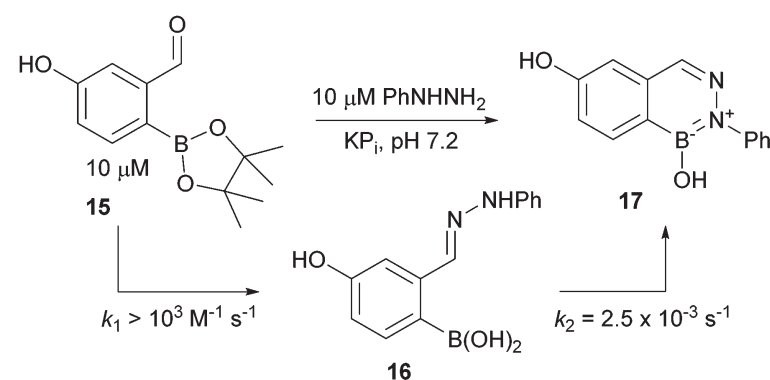

Fig. 4 BIQ formation proceeds at low concentration under mild conditions.

in aqueous conjugations. ${ }^{21}$ We present here our own exploration of the reaction, which is fully consistent with the reports of the Bane lab. We also include additional mechanistic data, new substrates, and the development of a fluorogenic process.

We began by examining the conjugation of compound $\mathbf{1 5}$ with phenylhydrazine in $\mathrm{pH} 7.2$ phosphate buffer. UV analysis could not provide the time resolution to determine the rate of hydrazone (16) formation but a lower bound of $>10^{3} \mathrm{M}^{-1} \mathrm{~s}^{-1}$ can be established. The cyclization step to deliver BIQ 17 was slower, with a rate constant of $2.5 \times 10^{-3} \mathrm{M}^{-1} \mathrm{~s}^{-1}$ (see Fig. 4). The rates were measured at $10 \mu \mathrm{M}$ concentration by following hydrazone formation and disappearance at $340 \mathrm{~nm}$. As expected, acidification increased the reaction rates, whereas under more basic conditions the transformations proceeded more slowly (see ESI†). We also found that polar aprotic solvents like DMF and MeCN drastically slowed down the BIQ formation and led to undesired oxidation of the boronic acid leaving a hydroxy substituted hydrazone. As a general rule we found that higher percentages of water increased the reaction rates even when the starting materials and products were poorly soluble. Complex media like human serum or millimolar concentrations of glutathione (see also ref. 21) only slightly affected the reaction rates (see ESI page 26†).

The relatively slow BIQ formation allowed us to employ NMR to follow the cyclization and to examine whether it was reversible. As shown in Fig. 5 arylhydrazone 16 cyclizes to BIQ 17 in aqueous phosphate buffer over the course of several hours - a rate that is consistent with the UV measurements. If purified product 17 is then treated with 2-FPBA (see panel B in Fig. 5), no change in the amount of $\mathbf{1 7}$ or $\mathbf{5}$ is observed over $24 \mathrm{~h}$. If 2-FPBA is added to $\mathbf{1 6}$ before cyclization is complete then small amounts of the oxime product are observed, indicating that arylhydrazone formation is reversible.

BIQ formation leads to an unusual aromatic scaffold whose optical properties might be amenable to tuning. Particularly interesting is the possibility that the de novo construction of<smiles>[Y7]O[B-]1c2ccc(O)cc2C(C)=N[N+]1(O)c1ccccc1</smiles>

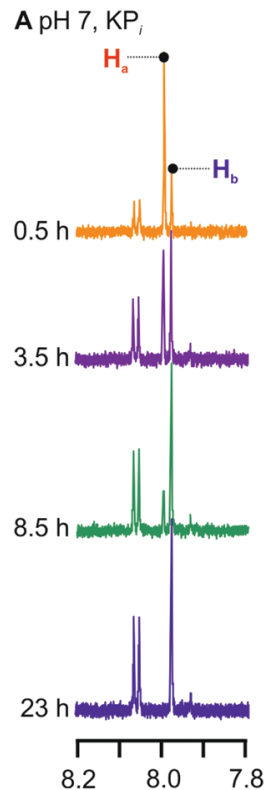

B 5 equiv 2-FPBA

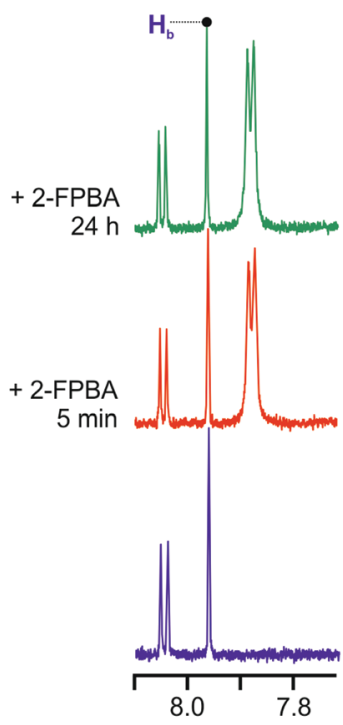

Fig. 5 NMR analysis of $B I Q$ formation (A) and reversibility (B). 
<smiles>[R]Cc1ccc2c(c1)C([R])=NN(c1ccc([R])cc1[R])[B-]2O</smiles>

$\mathrm{R}_{1}=\mathrm{R}_{2}=\mathrm{R}_{3}=\mathrm{R}_{4}=\mathrm{H}, 4$

$\mathrm{R}_{1}=\mathrm{R}_{3}=\mathrm{R}_{4}=\mathrm{H}, \mathrm{R}_{2}=\mathrm{Me}, 18$

$\mathrm{R}_{1}=\mathrm{OCH}_{2} \mathrm{CCH}, \mathrm{R}_{2}=\mathrm{R}_{3}=\mathrm{R}_{4}=\mathrm{H}, 19$

$\mathrm{R}_{1}=\mathrm{R}_{2}=\mathrm{R}_{3}=\mathrm{H}, \mathrm{R}_{4}=\mathrm{NO}_{2}, 20$

$R_{1}=R_{2}=H, R_{3}=N_{2}, R_{4}=O M e, 21$

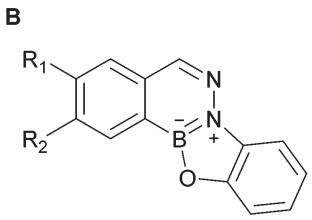

$$
\begin{aligned}
& \mathrm{R}_{1}=\mathrm{R}_{2}=\mathrm{H}, \mathbf{2 2} \\
& \mathrm{R}_{1}=\mathrm{OH}, \mathrm{R}_{2}=\mathrm{H}, 23 \\
& \mathrm{R}_{1}=\mathrm{H}, \mathrm{R}_{2}=\mathrm{OH}, 24 \\
& \mathrm{R}_{1}=O C \mathrm{OH}_{2} C \mathrm{CH}, \mathrm{R}_{2}=\mathrm{H}, 25 \\
& \mathrm{R}_{1}=\mathrm{H}, \mathrm{R}_{2}=\mathrm{OMe}, 26 \\
& \mathrm{R}_{1}=\mathrm{NMe}_{2}, \mathrm{R}_{2}=\mathrm{H}, 27
\end{aligned}
$$

Fig. 6 Representative examples of $\mathrm{BIQs}$ synthesized (see the $\mathrm{ESI} \dagger$ for additional examples and information).

the ring might allow turn-on fluorescence. The modularity of the reaction allowed us to quickly access a variety of substituted BIQs and study their absorbance and fluorescence. In general all tested BIQs showed some fluorescence at excitation wavelengths $<300 \mathrm{~nm}$, but most with an efficiency that would not be useful in practice (see panel A in Fig. 6 for structures and the ESI page $30 \dagger$ for absorbance and fluorescence spectra).

Two thoughts guided the design of our next series of BIQs: We considered the B-OH group as a potential fluorescence quencher and we also thought that additional annulation might red-shift the absorption wavelength. Accordingly, we synthesized another series of substituted BIQs (see panel b in Fig. 6) with an additional $O$-substituted five-membered ring (see as well the ESI $\dagger$ for an example of a six-membered ring). In general the alkoxy or hydroxyl substitutions did not lead to substantial shifts in absorbance maximum or improvements in fluorescence efficiency. Substitution with the electron rich dimethylamino group in the boronic acid component, however, delivered a good blue fluorophore (27 in Fig. 6). As shown in Fig. 7, the starting boronic acid, 28, was poorly fluo-

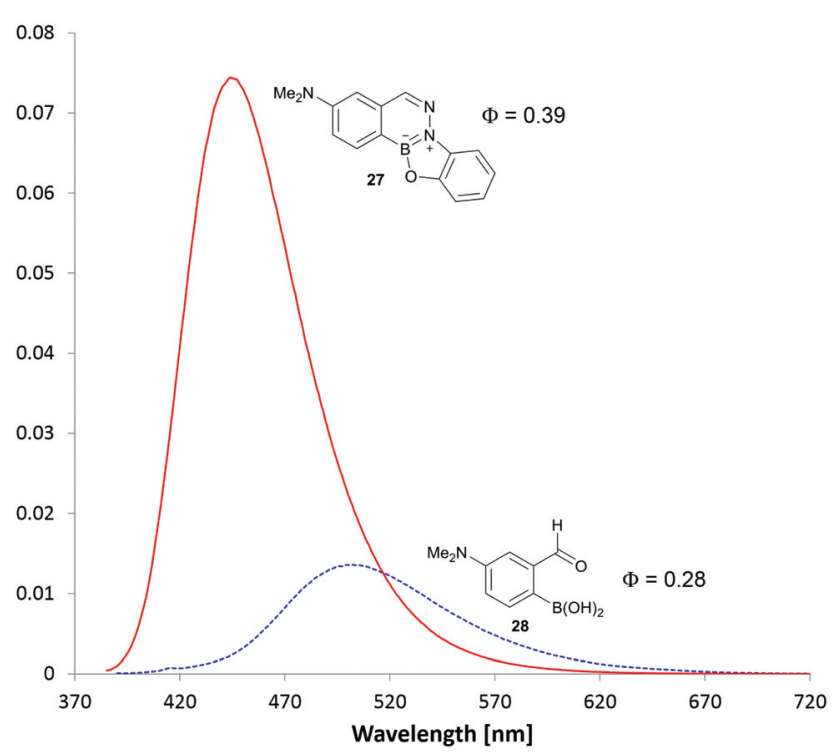

Fig. 7 Fluorescence enhancement upon BIQ formation. rescent with a maximum emission at $502 \mathrm{~nm}$. BIQ formation led to a more than five-fold increase in fluorescence and an improvement in fluorescent quantum yield $(\phi=0.39$ for 27 versus 0.28 for 28 ). Although improvements still need to be made for this fluorophore to service areas of unmet need in chemical biology, the fact that a fluorogenic reaction could be arrived at from a simple two-step optimization with less than fifteen compounds bodes well for future optimization.

\section{Conclusions}

We have presented mechanistic data and a comparison of strengths and weaknesses of various Schiff base reactions with $\alpha$-effect amines. Arylhydrazines are unique in that they undergo a secondary reaction to deliver aromatic BIQs that are highly stable. Optimization of substituents led to the development of a BIQ which exhibits a large fluorescence enhancement in comparison to its constituent components. Our studies should provide guidance for practitioners in chemical biology who would like to employ the process: acyl hydrazone and ketoxime formation is rapidly reversible and would likely be ideal in dynamic covalent chemistry; aldoximes are slowly reversible and might be ideally suited for applications where targeted slow release or exchange is desired; arylhydrazones lead to BIQ formation and would be ideal when the linkage is meant to be permanent.

\section{Acknowledgements}

Professor Oliver Wenger and Mr Angelo Lanzilotto are gratefully acknowledged for discussions on photochemistry and help in optical measurements. Mr Thomas Müntener is gratefully acknowledged for help with NMR measurements.

\section{Notes and references}

1 K. Lang and J. W. Chin, Chem. Rev., 2014, 114, 4764-4806.

2 D. M. Patterson, L. A. Nazarova and J. A. Prescher, ACS Chem. Biol., 2014, 9, 592-605.

3 N. Krall, F. P. da Cruz, O. Boutureira and G. J. L. Bernardes, Nat. Chem., 2015, 103-113.

4 P. Schmidt, L. Zhou, K. Tishinov, K. Zimmermann and D. Gillingham, Angew. Chem., Int. Ed., 2014, 53, 10928-10931.

5 E. T. Kool, D.-H. Park and P. Crisalli, J. Am. Chem. Soc., 2013, 135, 17663-17666.

6 P. Crisalli and E. T. Kool, Org. Lett., 2013, 15, 1646-1649.

7 P. Schmidt, C. Stress and D. Gillingham, Chem. Sci., 2015, 6, 3329-3333.

8 J. Kalia and R. T. Raines, Angew. Chem., Int. Ed., 2008, 120, 7633-7636.

9 A. Bandyopadhyay and J. Gao, Chem. - Eur. J., 2015, 21, 14748-14752. 
10 P. T. Corbett, J. Leclaire, L. Vial, K. R. West, J.-L. Wietor, J. K. M. Sanders and S. Otto, Chem. Rev., 2006, 106, 36523711.

11 E. M. Sletten and C. R. Bertozzi, Angew. Chem., Int. Ed., 2009, 48, 6974-6998.

12 P. Agarwal, J. van der Weijden, E. M. Sletten, D. Rabuka and C. R. Bertozzi, Proc. Natl. Acad. Sci. U. S. A., 2013, 110, 46-51.

13 M. J. S. Dewar and R. C. Dougherty, J. Am. Chem. Soc., 1964, 86, 433-436.

14 P. Tschampel and H. R. Snyder, J. Org. Chem., 1964, 29, 2168-2172.

15 P. D. Robinson, M. P. Groziak and L. Chen, Acta Crystallogr., Sect. C: Cryst. Struct. Commun., 1998, 54, 71-73.

16 M. P. Groziak, L. Chen, L. Yi and P. D. Robinson, J. Am. Chem. Soc., 1997, 119, 7817-7826.

17 P. D. Robinson and M. P. Groziak, Acta Crystallogr., Sect. C: Cryst. Struct. Commun., 1999, 55, 1701-1704.

18 D. Kanichar, L. Roppiyakuda, E. Kosmowska, M. A. Faust, K. P. Tran, F. Chow, E. Buglo, M. P. Groziak, E. A. Sarina, M. M. Olmstead, I. Silva and H. H. Xu, Chem. Biodiversity, 2014, 11, 1381-1397.
19 M. A. Grassberger, F. Turnowsky and J. Hildebrandt, J. Med. Chem., 1984, 27, 947-953.

20 M. C. Davis, S. G. Franzblau and A. R. Martin, Bioorg. Med. Chem. Lett., 1998, 8, 843-846.

21 O. Dilek, Z. Lei, K. Mukherjee and S. Bane, Chem. Commun., 2015, 51, 16992-16995.

22 P. Shieh and C. R. Bertozzi, Org. Biomol. Chem., 2014, 12, 9307-9320.

23 C. Le Droumaguet, C. Wang and Q. Wang, Chem. Soc. Rev., 2010, 39, 1233-1239.

24 B. E. Collins, S. Sorey, A. E. Hargrove, S. H. Shabbir, V. M. Lynch and E. V. Anslyn, J. Org. Chem., 2009, 74, 40554060 .

25 A. Adamczyk-Woźniak, K. M. Borys, I. D. Madura, A. Pawełko, E. Tomecka and K. Żukowski, New J. Chem., 2013, 37, 188-194.

26 J. Yan, G. Springsteen, S. Deeter and B. Wang, Tetrahedron, 2004, 60, 11205-11209.

27 P. M. S. D. Cal, J. B. Vicente, E. Pires, A. V. Coelho, L. s. F. Veiros, C. Cordeiro and P. M. P. Gois, J. Am. Chem. Soc., 2012, 134, 10299-10305.

28 A. J. J. Lennox and G. C. Lloyd-Jones, J. Am. Chem. Soc., 2012, 134, 7431-7441. 